\title{
Superconducting kinetic inductance photon detectors
}

Benjamin A. Mazin, Peter K. Day, Henry G. LeDuc, Anastasios Vayonakis, Jonas Zmuidzinas

Benjamin A. Mazin, Peter K. Day, Henry G. LeDuc, Anastasios Vayonakis, Jonas Zmuidzinas, "Superconducting kinetic inductance photon detectors," Proc. SPIE 4849, Highly Innovative Space Telescope Concepts, (18 December 2002); doi: 10.1117/12.460456

Event: Astronomical Telescopes and Instrumentation, 2002, Waikoloa, Hawai'i, United States 


\title{
Superconducting Kinetic Inductance Photon Detectors
}

\author{
Benjamin A. Mazin ${ }^{a}$, Peter K. Day ${ }^{b}$, Henry G. LeDuc ${ }^{b}$, \\ Anastasios Vayonakis ${ }^{a}$, and Jonas Zmuidzinas ${ }^{a}$ \\ ${ }^{a}$ California Institute of Technology, 1200 E. California Blvd., Pasadena, CA, 91125 \\ ${ }^{b}$ Jet Propulsion Laboratory, 4800 Oak Grove Drive, Pasadena, CA, 91109
}

\begin{abstract}
We are investigating a novel superconducting detector and readout method that could lead to photon counting, energy resolving focal plane arrays. This concept is intrinsically different from STJ and TES detectors, and in principle could deliver large pixel counts, high sensitivity, and Fano-limited spectral resolution in the optical/UV/X-ray bands. The readout uses the monotonic relation between the kinetic surface inductance $L_{s}$ of a superconductor and the density of quasiparticles $n$, which holds even at temperatures far below $T_{c}$. This allows a sensitive readout of the number of excess quasiparticles in the detector by monitoring the transmission phase of a resonant circuit. The most intriguing aspect of this concept is that passive frequency multiplexing could be used to read out $\sim 10^{4}$ detectors with a single HEMT amplifier. Single x-ray events have been observed in prototype detectors.
\end{abstract}

Keywords: Superconducting, Detectors, Kinetic Inductance, Cryogenic, STJ, TES, KID

\section{INTRODUCTION}

Energy resolving detectors possess unique qualities which will likely make them a very important technology for many fields of astronomy. The small band gap associated with low temperature superconductors may be used to measure the energy of incident photons. Current devices undergoing development for the optical/UV include superconducting tunnel junctions (STJ), transition edge sensors (TES), and kinetic inductance detectors (KIDs). Unlike conventional CCDs and microchannel plate detectors, these devices promise a very wide bandwidth, moderate resolution spectroscopy, and photon counting. These benefits currently come at the cost of low pixel counts and expensive, complex cooling systems.

Small pixel format arrays of superconducting detectors will enable unique science from the ground and from space. The initial targets in the optical/UV will be point sources with interesting time variability, such as pulsars, ${ }^{1}$ magnetic white dwarfs ${ }^{2}$ and low mass X-ray binaries. X-ray science with medium-sized arrays include such exciting missions as Constellation-X. ${ }^{3}$ Larger format arrays will enable a host of interesting projects. Calculations have shown that a survey performed with an energy resolving focal plane can be more than ten times as efficient as a conventional survey with CCDs and filters. ${ }^{4}$

A particularly exciting application for medium format (256x256 pixel) energy resolving detectors is the Terrestrial Planet Finder. For the optical coronograph form of TPF, an energy resolving focal plane will allow the mirror tolerances to be relaxed significantly, perhaps by a factor of $\sim 3 .^{5}$ This is due to the fact that the speckles from diffracted and scattered light will move with wavelength while the light signature of planets will not. The inherent energy resolution of the detectors will do away with the need for a separate spectrometer and filters, making for a more sensitive instrument for a given mirror size. This addresses one of the serious problems with TPF - what if there are no terrestrial planets in the closest $\sim 10^{2}$ stars systems that a 10 meter coronograph can cover in a reasonable amount of time? Increasing the sensitivity allows more systems to be surveyed in a given amount of time, allowing a greater possibility of success. Since the largest technical hurdles for TPF are the mirror tolerances and the small number of star systems to be surveyed, superconducting detectors should be considered a very important technology for this mission. 


\section{DEVICE CONCEPT}

Our detector, the kinetic inductance detector, uses a novel method to read out the energy of photons absorbed by a superconductor. The absorbed photon breaks Cooper pairs, creating quasiparticle excitations. The quasiparticles block Cooper pairs from occupying some of the electron states (through the exclusion principle). Quasiparticles therefore affect the complex (a.c.) electrical conductivity, and thus the surface impedance $Z_{s}$. The surface impedance of superconductors is primarily inductive; this is the "kinetic inductance".

The properties of thin-film superconducting transmission lines are modified by the kinetic inductance. The magnitude of this effect is controlled by a dimensionless parameter $\alpha=\lambda / w_{\text {eff }}$. Here $\lambda$ is the magnetic penetration depth, determined by the surface reactance $X_{s}=\mu_{0} \omega \lambda$, and $w_{\text {eff }}$ is a scale length which characterizes the spatial extent of the fields in the transmission line. In particular, the phase velocity is reduced by the factor $\sqrt{1+\alpha}$, which depends on the quasiparticle density through $X_{s}$.

An obvious way to measure this effect is to design a superconducting transmission line resonator and measure the transmission phase of a microwave probe signal at the resonant frequency, $f_{0}$, of the device. A photon absorbed by the resonator will result in a change in the transmission phase that is proportional to the photon energy. This sudden change in phase will gradually decay back to the original value as the quasiparticles recombine. The timescale of this recombination, $\tau_{q p}$, varies with material and temperature and can range from microseconds to milliseconds. This timescale sets the maximum count rate of the detector. The phase pulse has a finite rise time which is governed by the resonator "ring-down" time, $\tau_{r}=Q_{r} / 2 \pi f_{0}$, where $Q_{r}$ is the resonator quality factor. This description holds for the case that $\tau_{r}<<\tau_{q p}$, which includes all the devices we have tested so far. For very high $Q_{r}$, it is possible to have $\tau_{q p}<<\tau_{r}$. In this case, the phase pulse rise time will be set by $\tau_{q p}$, and the fall time by $\tau_{r}$. In fact, it is possible to avoid the "falling" portion of the phase waveform entirely, by adjusting the phase of the microwave source appropriately.

The primary advantage of this scheme is that passive frequency multiplexing can be achieved by using lithography to set slightly different resonant frequencies for many resonators connected to a single transmission line. Since each resonator only responds at its resonant frequency, it is possible to read out all the devices simultaneously using a single cryogenic HEMT amplifier. This should allow more that $10^{3}$ devices to be read out through one amplifier. Unlike TESs, which require cryogenic electronics (SQUIDs), this passive frequency multiplexing removes all the complexity from the cold stage and transfers it to the room temperature electronics of the readout. The tremendous recent advances in microwave technology make a readout for a large array feasible.

\section{CPW RESONATOR DESIGN AND PREDICTED BEHAVIOR}

To build an optimal detector we must first understand the relevant physics. To this end, we will briefly touch on the applicable BCS theory and describe one of the simplest examples of a KID, a coplanar waveguide (CPW) transmission line resonator. ${ }^{6}$ More details can be found in reference. ${ }^{7}$

A half-wave CPW resonator consists of a section of CPW transmission line with gap capacitors on both ends, as shown in Fig. 1. The device we will analyze in this paper has a $5 \mu \mathrm{m}$ center strip, $11 \mu \mathrm{m}$ spacing between ground planes in order to get a $50 \Omega$ transmission line, $2 \mu \mathrm{m}$ gap capacitors, and a length of $12 \mathrm{~mm}$. The thickness of the aluminum film is $2200 \AA$. On sapphire with a dielectric constant $\epsilon \approx 10$, this yields a first resonance at $\sim 5 \mathrm{GHz}$, according to:

$$
f_{0}=\frac{n c}{l \sqrt{2(1+\epsilon)}}
$$

where $n$ is the mode number and $l$ is the length of the resonator.

The responsivity of a properly designed KID, measured in degrees of phase shift per quasiparticle, is governed by two main factors - the volume of superconductor and the quality factor $Q_{r}$ of the resonator. This quality

factor $Q_{r}$ is determined by the various energy loss mechanisms in the resonator - ohmic loss, $Q_{m}$, resonator loading due to energy leaking out through the coupling capacitors, $Q_{c}$, and radiation loss, $Q_{\text {rad }}$. 


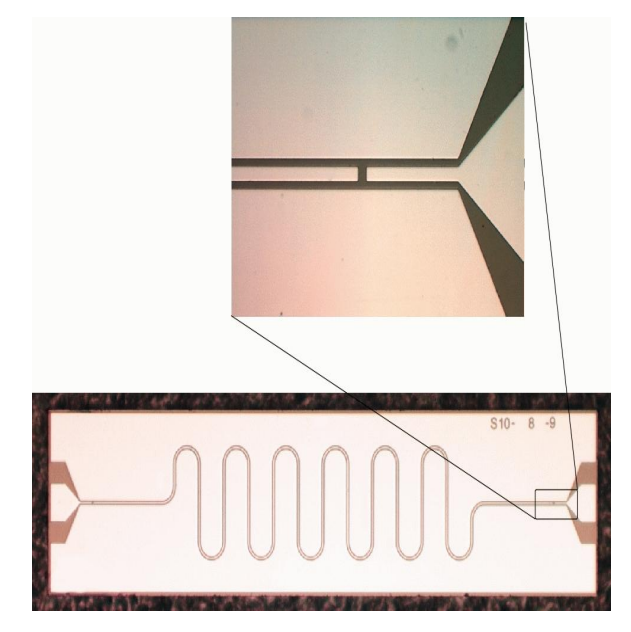

Figure 1. Layout of a CPW half-wave resonator with a $10 \mu \mathrm{m}$ center strip, $22 \mu \mathrm{m}$ spacing between ground planes, $8 \mu \mathrm{m}$ capacitors, and a length of $9 \mathrm{~mm}$.

In theory, ohmic loss is due to dissipation by quasiparticles. The Mattis-Bardeen ${ }^{8}$ theory of the electrodynamics of BCS superconductors may be used to calculate $Q_{m}$. At higher temperatures, where there is a higher population of quasiparticles, $Q_{m}$ will limit $Q_{r} \approx Q_{m}$.

In the limit $k_{B} T<<\Delta(0), \hbar \omega<<\Delta(0)$, the imaginary part of the conductivity can be approximated as

$$
\frac{\sigma_{2}}{\sigma_{n}} \approx \frac{\pi \Delta(T)}{\hbar \omega}\left[1-\exp \left(-\Delta(0) / k_{B} T\right) \exp \left(-\hbar \omega / 2 k_{B} T\right) I_{0}\left(\hbar \omega / 2 k_{B} T\right)\right]
$$

where $I_{0}(x)$ is a modified Bessel function. This expression holds regardless of whether the photon energy $\hbar \omega$ is large or small compared to the thermal energy $k_{B} T$. The real part of the conductivity, which is responsible for the loss, and is due to thermally-excited quasiparticles, is given by a similar expression in this limit:

$$
\frac{\sigma_{1}}{\sigma_{n}} \approx \frac{2 \Delta(T)}{\hbar \omega} \exp \left(-\Delta(0) / k_{B} T\right) \exp \left(\hbar \omega / 2 k_{B} T\right) K_{0}\left(\hbar \omega / 2 k_{B} T\right)\left(1-\exp \left(-\hbar \omega / 2 k_{B} T\right)\right)
$$

Here $K_{0}(x)$ is also a modified Bessel function. $Q_{m}$ can be evaluated directly from these expressions

$$
Q_{m}=\frac{-1}{\alpha \gamma} \frac{\sigma_{2}}{\sigma_{1}}=\frac{-1}{\alpha \gamma} Q_{\sigma}
$$

where $\alpha$ is the fraction of the total inductance of the transmission line due to kinetic inductance described above, and $\gamma$ relates a change in the surface impedance to a change in conductivity:

$$
\frac{\delta Z_{s}}{Z_{s}}=-\gamma \frac{\delta \sigma}{\sigma}
$$

It is possible to calculate $\alpha$ for our geometry using by using a finite element calculation that minimizes the sum of the magnetic field energy and the electron kinetic energy to find the current distribution in the superconducting transmission line. ${ }^{9}$ For this resonator we calculate $\alpha=0.055$. The coefficient $\gamma$ should be between -0.5 and -1 depending on the thickness of the superconducting film and the penetration depth. Since we expect the penetration depth to be around $500 \AA$ and our film thickness is $2200 \AA$, we expect $\gamma$ to be close to -0.5 .

The coupling loss, $Q_{c}$, is due to energy leaking out of the resonator through the capacitors at each end of the resonator. The coupling loss may be calculated using a numerical electromagnetic field simulator such as Sonnet. ${ }^{10}$ The leakage through the capacitor, $S_{21}$, is used to calculate $Q_{c}$ : 


$$
Q_{c}=\frac{\pi}{\left|S_{21}\right|^{2}}
$$

$Q_{c}$ can be made arbitrarily large by using capacitors with larger gaps, so should never be a limiting factor in a properly designed resonator. However, resonators limited by $Q_{c}$ have the nice property of being over-coupled, yielding near perfect transmission on resonance.

The radiation loss, $Q_{\text {rad }}$, can be approximated by calculating the radiation produced by a straight transmission line on a semi-infinite dielectric substrate. We find that for a half-wave CPW resonator on sapphire,

$$
Q_{\text {rad }}=3.4\left(\frac{L}{s}\right)^{2}
$$

where $L$ is the length of the resonator and $s$ is the distance between CPW ground planes. This calculation is not accurate due to the approximations that have been made, but it gives a rough estimate. For the device we are analyzing, $Q_{c}=2.9 \times 10^{6}$ and $Q_{r a d}=7.7 \times 10^{6}$. For aluminum at our base temperature of $60 \mathrm{mK}, Q_{m}$ is essentially infinite. This means our resonator should be coupling limited with a predicted $\frac{1}{Q_{r}}=\frac{1}{Q_{c}}+\frac{1}{Q_{\text {rad }}}$, or $Q_{r}=2.1 \times 10^{6}$

The change in resonant frequency of the resonator can also be described by similar equations. The resonant frequency $f_{0}$ varies with temperature as:

$$
\begin{gathered}
f_{0}=f_{0}(0)\left[1-\frac{\alpha}{2} \frac{\delta X_{s}(T)}{X_{s}(0)}\right] \\
\delta X_{s}(T)=X_{s}(T)-X_{s}(0)
\end{gathered}
$$

where $X_{s}$ is the surface impedance of the superconductor. Combining these equations yields an expression for the change in resonant frequency with temperature:

$$
f_{0}=f_{0}(0)\left[1+\frac{\alpha \gamma}{2} \frac{\delta \sigma_{2}(T)}{\sigma_{2}(0)}\right]
$$

In reality there are several other effects to consider when determining the ultimate performance of a given design, including amplifier and electronics noise, power handling capability of the resonator, external quasiparticle sources (pair-breaking radiation, etc.), and magnetic fields/trapped flux. These issues, while often important, are beyond the scope of this summary. More information can be found here. ${ }^{7}$

\section{RESONATOR CHARACTERIZATION}

The predicted behavior of this resonator with temperature can be compared with experimental data. The resonator was mounted into a small sample block and wire bonded to coaxial to CPW transitions. The block was then tested in a dilution refrigerator at various temperatures. A low noise, broadband HEMT amplifier with a noise temperature of around $20 \mathrm{~K}$ from $2-20 \mathrm{GHz}$ was mounted in the dilution refrigerator at $4 \mathrm{~K}$ as a first stage amplifier. Room temperature amplifiers with higher noise temperatures were used to further boost the signal. The device was measured using a HP 8720 network analyzer, yielding the raw data shown in Fig. 3 .

The data from the network analyzer consists of the complex transmission amplitude $S_{21}$ for 1601 frequency points in the desired interval. This data are calibrated as the top of the dewar, so losses in the semi-rigid coax and gains from the cryogenic amplifiers are not included, but can be estimated.

This complex data set is then fit to a resonance model that includes the expected resonance and various leakage paths that act in parallel with the main resonant transmission mode. The model is shown in equation 12 . 


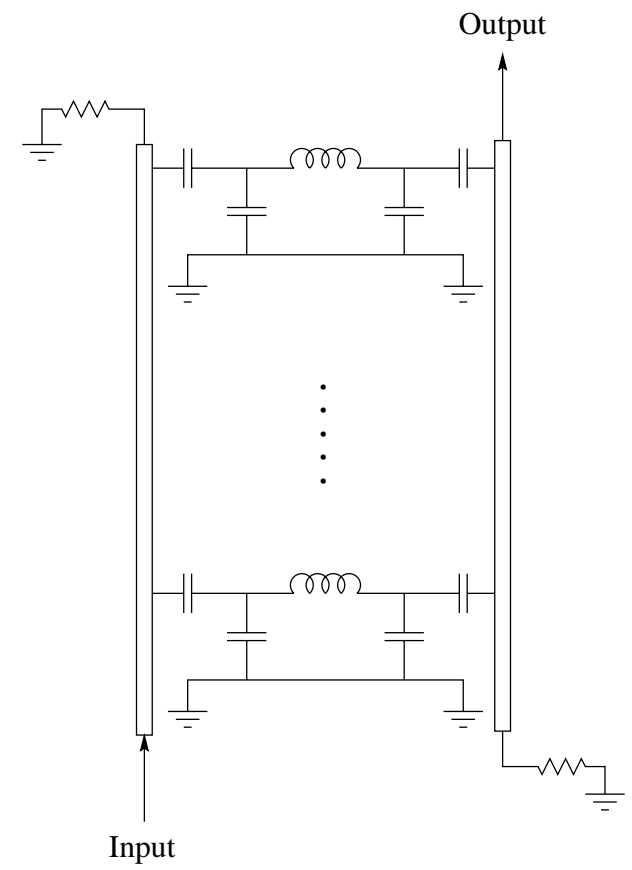

Figure 2. A possible multiplexing scheme.

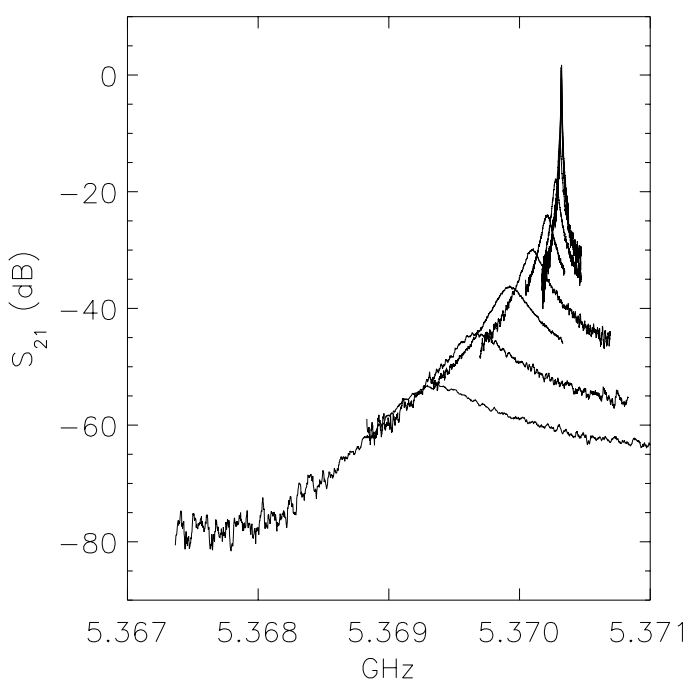

Figure 3. Smoothed data from the aluminum resonator described above. Various temperatures are overlayed to show the sharpening of the resonance as the temperature is lowered. The broadest resonance is $400 \mathrm{mK}$, the sharpest is 60 mK. 

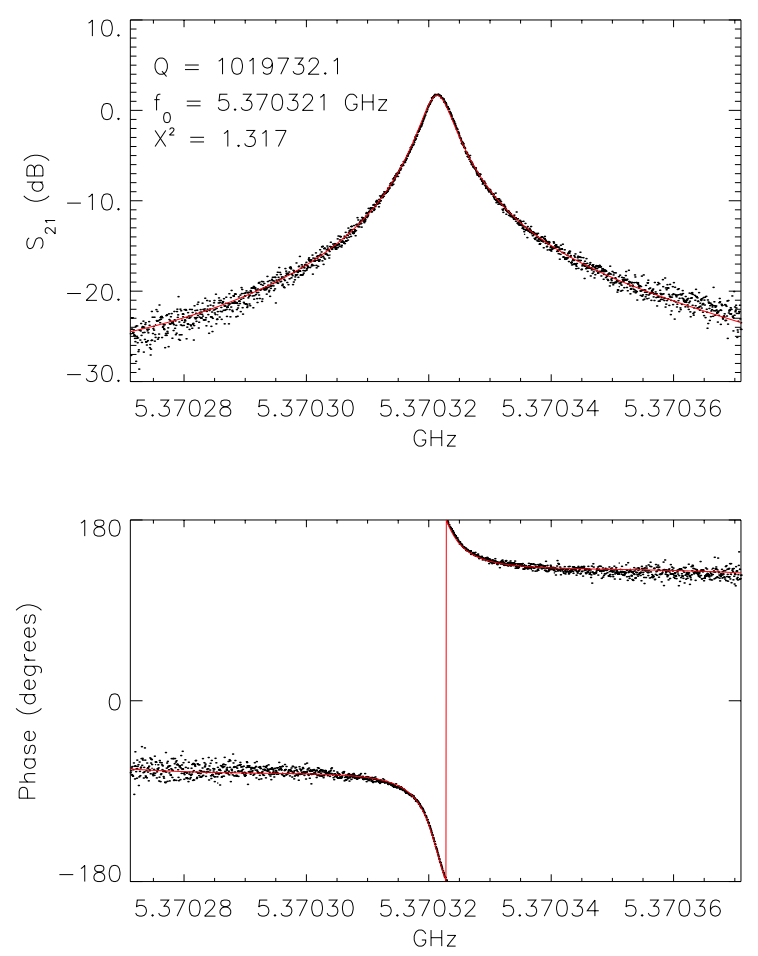

Figure 4. Resonator data and fit for aluminum resonator described above at $T=60 \mathrm{mK}$.

$$
\begin{gathered}
\delta x=\frac{f-f_{0}}{f_{0}} \\
S_{21_{\text {data }}}=\frac{S_{21_{\text {max }}}}{1+i 2 Q_{r} \delta x}+S_{21_{\text {leak }}}
\end{gathered}
$$

where the fitting parameters are $Q_{r}, f_{0}$, the real and imaginary part of the maximum transmitted resonance signal, $S_{21_{\text {max }}}$, the real and imaginary part of the leakage, $S_{21_{l e a k}}$, and terms to compensate for the slope of the leakage magnitude and phase. The slope parameters, while important for a good fit, are small and not particularly important for the determination of $Q_{r}$ and $f_{0}$, the primary parameters of interest. The fit to the lowest temperature data can be seen in Fig. 4.

The data shows that the resonance increases to a $Q_{r}$ of around one million at low temperatures. The transmission through the sample at resonance is around $0 \mathrm{~dB}$ when corrected for expected throughput changes with temperature, which means that this device is coupling limited, as expected.

Using the equations given in Section 3, we can fit the temperature dependence of the resonator using three parameters: the superconducting gap at $T=0, \Delta(0)$; the fraction of total inductance contributed by the kinetic inductance $\alpha$; and $\gamma$, which connects changes in the conductivity to changes in surface impedance. The parameters $\alpha$ and $\gamma$ are degenerate, so we set the value of $\alpha$ derived from the numerical field simulations, 0.055 , in order to get a value for $\gamma$.

Fitting both $Q_{r}$ and $f_{0}$ vs. $T / T_{c}$ simultaneously with error bars derived from the $S_{21}$ fits, the drift specification of the network analyzer's crystal frequency reference, and the temperature uncertainty yields the values shown in the upper right corner of Fig. 5. These values are quite reasonable, but the reduced $\chi^{2}$ value of 5.04 shows that some of the data points deviate from theory. 

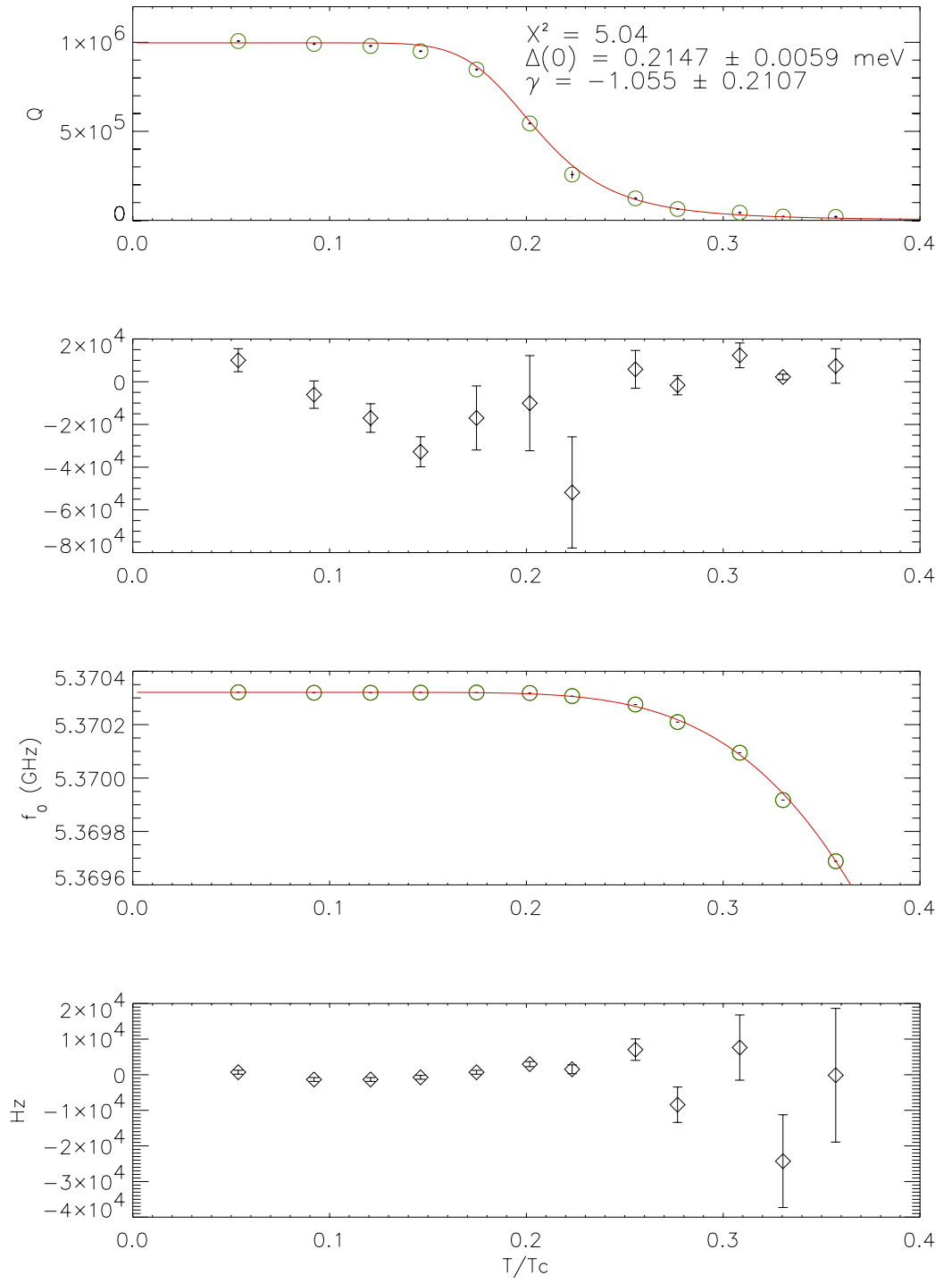

Figure 5. $Q$ and $f_{0}$ fits of the resonator data from the aluminum on sapphire, $5 \mu \mathrm{m}$ center strip, $12 \mathrm{~mm}$ long CPW resonator. 


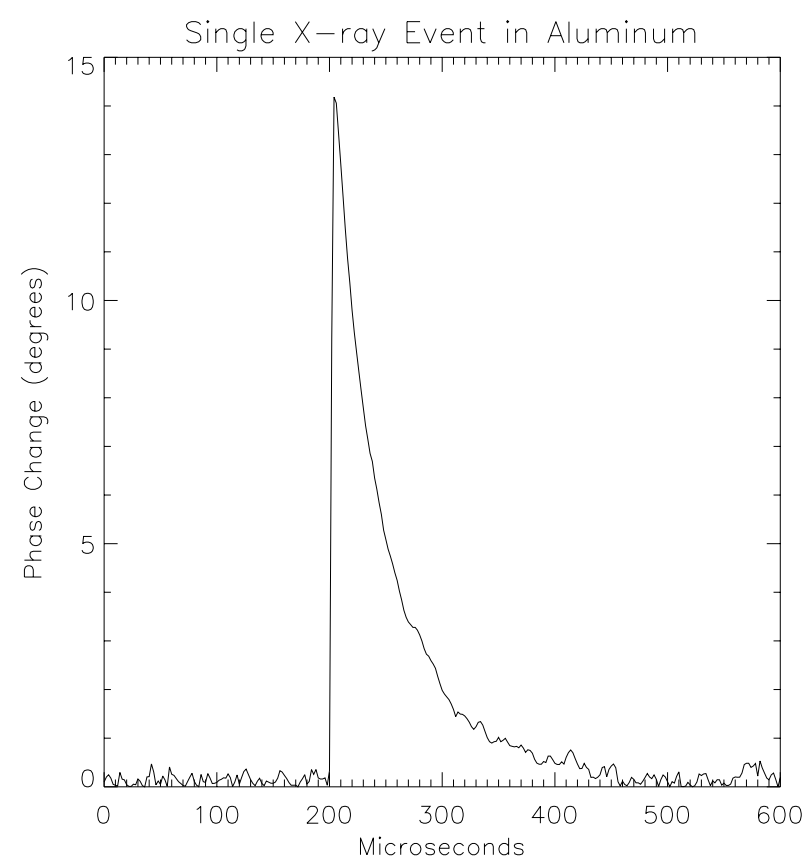

Figure 6. Phase pulse from a $5.9 \mathrm{keV}$ x-ray in an aluminum resonator with $Q \approx 50000$.

This aluminum device matches the theory fairly well. There are hints of departures from the simple theory, but more data needs to be collected and analyzed before we can come to any firm conclusions. It is possible that some of the deviations can be explained by magnetic flux trapped in the film - future experiments will utilize a magnetic shield to eliminate this potential problem.

\section{PHOTON DETECTION}

The data presented so far has been steady state. In order to test these devices as detectors we introduced two sources of pair breaking radiation, an iron-55 source that produces $5.9 \mathrm{keV}$ and $6.1 \mathrm{keV}$ x-rays and an optical fiber that delivers pulses of red photons generated by an LED. The data we present here is from a larger CPW resonator than the one described in Section 2, with a much lower $Q_{r}(\sim 50,000)$.

\subsection{Pulse Readout}

Many different pulse readouts are possible for these resonators. For the experiments described here we use a very simple scheme. A microwave signal is generated at room temperature, tuned to the resonant frequency of the device, and sent to the resonator. The output of the resonator passes through a cryogenic HEMT amplifier, and then through a room temperature amplifier chain. This amplified signal is then mixed with a copy of the original signal in an IQ mixer. This yields two outputs, I and Q, the real and imaginary parts of the probe signal. The angle, $\phi=\tan ^{-1}(I / Q)$, is the phase. We amplify, filter, and digitize I and $\mathrm{Q}$, and reconstruct the phase output with a sampling interval of 1 microsecond.

\subsection{Results}

Due to the sinusoidal shape of the current distribution in our resonators, we expect the response to monochromatic light to exhibit a positional dependence. This positional dependence is the cause of the low energy tails on the x-ray distribution. The red LED pulses illuminate the entire detector with a large number of photons, and therefore show no positional dependence. The histogram in Fig. 7 shows that this device exhibits an energy resolution, $R=E / \delta E$, of about $R \sim 30$ for the light pulses, lower than one would expect from Poisson statistics 


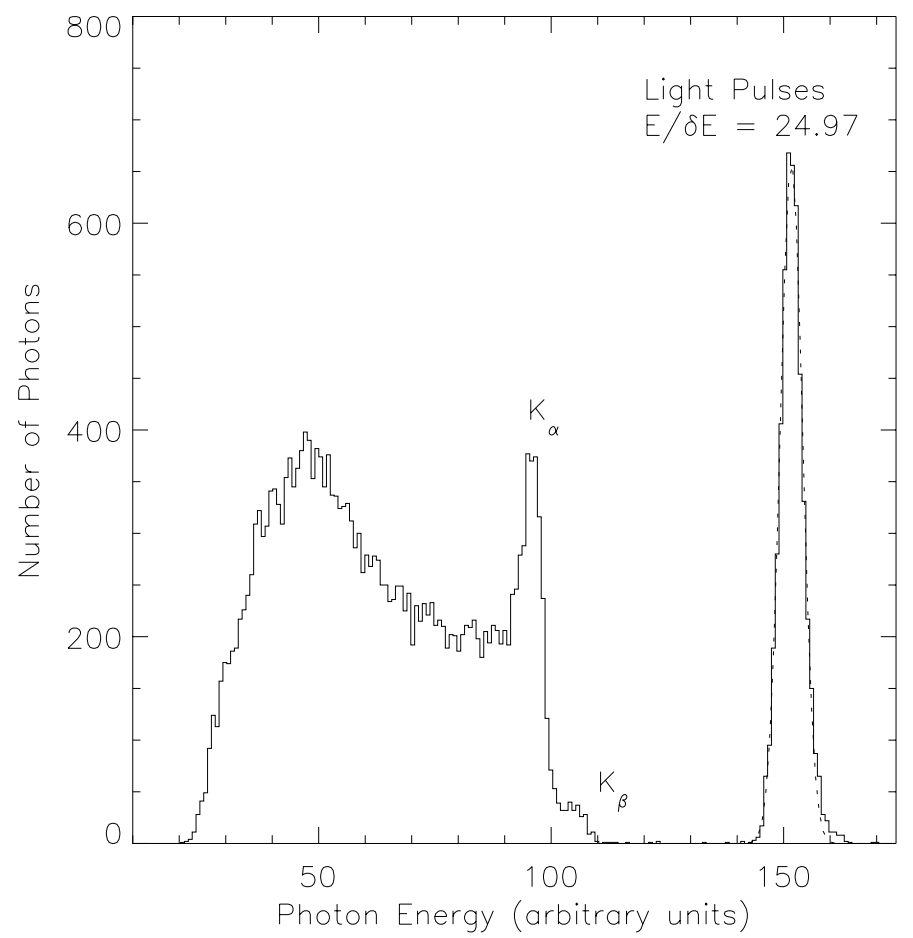

Figure 7. Histogram of phase pulses from an iron- 55 x-ray source and red LED in an aluminum resonator with $Q \sim 50000$. The superconductor initially absorbs an energy of $\sim 10 \mathrm{keV}$ from each light pulse, so they lie to the right of the $6 \mathrm{keV}$ $\mathrm{x}$-ray pulses on this plot.

of the photon pulse. Fits to the x-ray distribuition show that the x-rays also display an $R \sim 30$. The signal to noise ratio shown in the pulse in Fig. 6 appears to be better than this, so we are currently investigating other sources of noise which are broadening the energy resolution.

\subsection{Multiplexing}

Multiplexing a resonator like the one described above is rather simple. The length of each resonator is simply varied to yield slightly different resonant frequencies, then the devices are strung like beads on a string, as seen in Fig. 2, which shows the equivalent circuit for a multiplexed lumped element resonator.

\section{DISCUSSION}

\subsection{Designing Actual Detectors}

The resonators described so far are not ideal detectors is any sense - they have a small active area and low fill factor, low QE, have a positional dependence, and are rather large. However, they fulfill their purpose as test devices quite nicely, since their very simple structure (one patterned superconducting film on a high-quality substrate) makes them easy to fabricate and analyze.

We have designed several different detectors which address these issues. The geometries considered include strip line resonators, quarter-wave resonator (see Fig. 8), and lumped element microstrip resonators, some utilizing dielectric films and bandgap engineering to increase performance. These designs can offer high fill factors, good sensitivity, and multiplexing. Further experimentation is required to determine which design will ultimately prove superior for the optical/UV. 


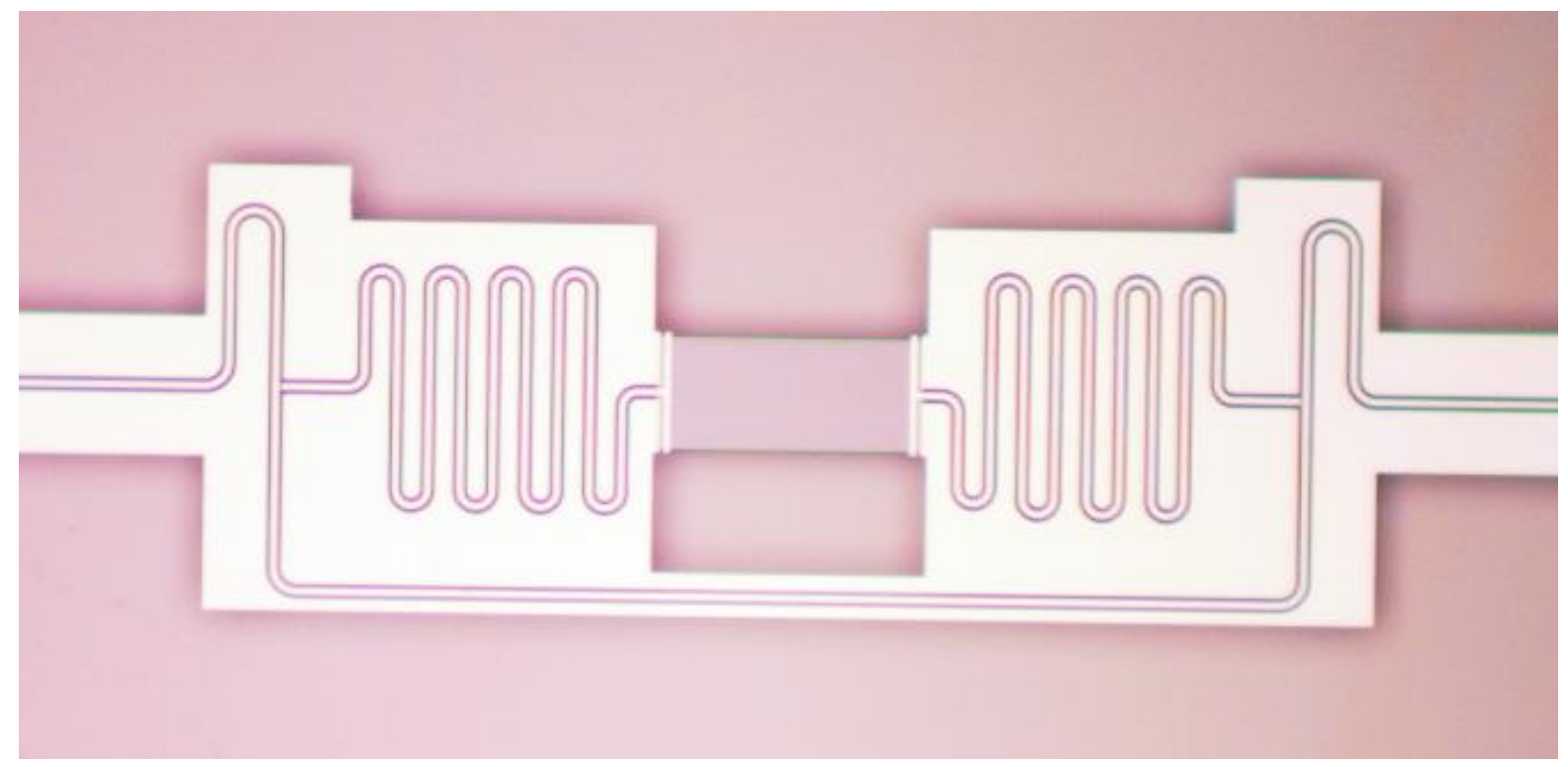

Figure 8. A position sensitive x-ray detector based on two aluminum CPW resonators at the either end of a tantalum absorber.

\subsection{Comparison to Existing Technology}

It is important to compare KIDs to the current state of the art in superconducting detectors, STJs and TESs.

STJs are the most similar to KIDs as they work at temperatures well below $T_{c}$. STJs have demonstrated energy resolutions of around 10 at $3000 \AA$ in arrays of up to 36 pixels ${ }^{11}{ }^{2}$ The real difficulties come when trying to scale the technology up. For large arrays, wiring problems, junction fabrication, and simultaneous magnetic biasing become important and difficult issues. The ESA STJ group has plans to build a camera with around one hundred pixels within the next several years. ${ }^{12}$ This is the largest format STJ array that we are aware of.

TESs are quickly becoming the energy resolving detectors of choice, as they have demonstrated the best energy resolution at optical wavelengths. ${ }^{13}$ Groups at Stanford, NIST, and Goddard are driving development and frequency or time division multiplexing schemes may allow multiplexing of 32 pixels per SQUID. ${ }^{14}$ TESs, even with good multiplexing, still present significant challenges. TES pixels can be quite small $5 \times 5 \mu \mathrm{m}$, and SQUIDS are quite large (at least $100 \times 100 \mu \mathrm{m}$ ). This can lead to low filling factors or wiring issues. Other factors include heat load from warmer SQUIDS, and complex cryogenic electronics. The largest TES device currently proposed is SCUBA-2 at 12,800 pixels. ${ }^{15}$ This many pixels requires a multi-million dollar development effort and is likely near the maximum number of pixels feasible with current technology.

Our kinetic inductance detectors should be much easier to scale to larger arrays. Using modest estimates, we believe over 1000 devices can be read out using one amplifier and electronics chain. If strip detectors are used, we envision a minimum of 500 strips of 30 pixels each for a total of 15000 pixels per amplifier, compared to at most 32 for a TES or around 15 for a STJ. This base unit of 15000 pixels can be scaled up by adding parallel chains.

\section{ACKNOWLEDGMENTS}

We would like to thank NASA Grant NAG5-10358, the JPL Director's Discretionary Fund, and the Caltech President's Fund for supporting this research. 


\section{REFERENCES}

1. R. Romani, A. Miller, B. Cabrera, S. Nam, and J. Martinis, "Phase-resolved crab studies with a cryogenic transition-edge sensor spectrophotometer," Ap. J. 563, pp. 221-228, Dec. 2001.

2. M. Perryman, M. Cropper, G. Ramsay, F. Favata, A. Peacock, N. Rando, and A. Reynolds, "High-speed energy-resolved stj photometry of the eclipsing binary uz for," Mon. Not. Roy. Astron. Soc. 324, pp. 899909, July 2001.

3. K. Weaver, N. White, H. Tananbaum, A. Valinia, F. Marshall, J. Bookbinder, C. Stahle, J. Grady, R. Rasche, L. Van Speybroeck, R. Kelley, S. Kahn, F. Harrison, and S. Castles, "Key technology development for constellation-x," Astron. \& Ap. (Suppl.) \#88.03 192, 1999.

4. B. Mazin and R. Brunner, "Simulated extragalactic observations with a cryogenic imaging spectrophotometer," Astron. J. 120, pp. 2721-2729, Nov. 2000.

5. W. Traub, "Private communication," 2002.

6. B. Mazin, P. Day, J. Zmuidzinas, and H. LeDuc, "Multiplexable kinetic inductance arrays," AIP Conference Proceedings 605, pp. 309-312, July 2001.

7. B. Mazin, P. Day, J. Zmuidzinas, H. LeDuc, and A. Vayonakis, "Multiplexable kinetic inductance detectors," 2003. (in preparation).

8. D. C. Mattis and J. Bardeen, "Theory of the anomalous skin effect in normal and superconducting metals," Phys. Rev. 111, pp. 412-417, 1958.

9. W. Chang, "Numerical-calculation of the inductances of a multisuperconductor transmission-line system," IEEE Trans. Magnetics 17, pp. 764-766, 1981.

10. Sonnet User's Manual Release 6.0, Sonnet Software, Inc., Liverpool, NY, 1999.

11. C. Wilson, K. Segall, L. Frunzio, L. Li, D. Prober, D. Schiminovich, B. Mazin, C. Martin, and R. Vasquez, "Optical/uv single-photon imaging spectrometers using superconducting tunnel junctions," Nucl. Instrum. Meth. A 444, pp. 449-452, Apr. 2000.

12. P. Verhoeve, "Superconducting tunnel junction detectors for ground-based optical astronomy and spacebased uv and x-ray astrophysics," AIP Conference Proceedings 605, pp. 559-564, July 2001.

13. A. Miller, B. Cabrera, R. Romani, E. Figueroa-Feliciano, S. Nam, and R. Clarke, "Development of wideband, time and energy resolving, optical photon detectors with application to imaging astronomy," Nucl. Instrum. Meth. A 444, pp. 445-448, Apr. 2000.

14. J. Staguhn and ret.al., "Tes detector noise limited readout using squid multiplexers," AIP Conference Proceedings 605, pp. 321-324, July 2001.

15. W. Duncan and ret.al., "Scuba-2: Application of ltd technology," AIP Conference Proceedings 605, pp. 577580, July 2001. 